\title{
CÁNCER EN PERSONAS QUE VIVEN CON VIH-SIDA EN UN HOSPITAL PÚBLICO DE REFERENCIA EN PERÚ
}

\author{
Lucía Maryelena Mendoza-Mori(1),a, Jim Brian Valenzuela-Medina(1),a, \\ Eduardo Gotuzzo (1,b, Fernando Alonso Mejía-Cordero (1,b, Elsa Violeta González-Lagos (1,c \\ 1 Instituto de Medicina Tropical “Alexander von Humboldt”, Universidad Peruana Cayetano Heredia, Lima, Perú. \\ a Médico cirujano, b médico especialista en enfermedades infecciosas y tropicales, ${ }^{c}$ médica cirujana, magíster en \\ Epidemiología Clínica
}

\section{RESUMEN}

El objetivo del estudio fue describir las características demográficas, clínicas y terapéuticas de pacientes con VIH-SIDA que desarrollaron algún tipo de cáncer. Se identificaron 276 casos de cáncer diagnosticados en el Hospital Nacional Cayetano Heredia entre el 2000 y 2018. El 80,8\% (223/276) correspondieron a neoplasias definitorias de sida (NDS), siendo el más frecuente el sarcoma de Kaposi; mientras que, entre las neoplasias no definitorias de sida (NNDS), el más frecuente fue el linfoma de Hodgkin. La mediana de edad fue 36,5 años, siendo más alta entre los casos diagnosticados de NNDS. Con respecto al nivel de linfocitos CD4 al diagnóstico de cáncer, la mediana entre las NDS fue mucho menor que las NNDS, 87,5 células/ $\mu \mathrm{L}$ y 216 células/ $\mu \mathrm{L}$, respectivamente. Por tanto, las NNDS tuvieron una historia más larga de infección por VIH, y una edad más avanzada al diagnóstico de cáncer, así como niveles de células CD4 más altos.

Palabras clave: VIH; SIDA; Neoplasias; América Latina. (fuente: DeCS BIREME).

\section{CANCER IN PEOPLE LIVING WITH HIV-AIDS AT A REFERRAL HOSPITAL IN LIMA, PERU}

\begin{abstract}
This study was carried out to describe and compare the demographic, clinical, and therapeutic characteristics of HIV patients who developed some cancer. We identified 276 cancer cases diagnosed at Hospital Cayetano Heredia between 2000 and 2018. 80,8\% (223/276) had AIDS-defining-cancers (ADCs), being Kaposi's Sarcoma the most frequent type; meanwhile, among non-AIDS-defining-cancers (NADCs), the most frequent was Hodgkin lymphoma. The median age was 36,5 years, being highest among the cases diagnosed with NADCs. Concerning CD4 lymphocyte counts, the median among ADCs was much lower than NADCs, 87,5 cells/ $\mu$ l and 216 cells/ $\mu$ l, respectively. Therefore, NADCs cases have a longer history of HIV infection, and an older age at cancer diagnosis, as well as higher CD4 cells counts.
\end{abstract}

Keywords: HIV, AIDS, Neoplasms, Latin America (source: MeSH NLM).

\section{INTRODUCCIÓN}

Citar como: Mendoza-Mori LM, Valenzuela-Medina JB, Gotuzzo E, Mejía-Cordero FA, González-Lagos EV. Cáncer en personas que viven con VIH-SIDA en un hospital público de referencia en Perú. Rev Peru Med Exp Salud Publica. 2021;38(2):27883. doi: https://doi.org/10.17843/ rpmesp.2021.382.6341

Correspondencia: Lucía Maryelena Mendoza Mori; lucia.mendoza.m@ upch.pe

Recibido: $13 / 08 / 2020$ Aprobado: $12 / 03 / 2021$ En Línea: 04/06/2021
El riesgo de desarrollar cáncer en las personas que viven con el VIH (PVV) es mayor que el de la población general; esto como consecuencia de la inmunosupresión causada por este virus, lo cual perjudica el control de las infecciones virales con potencial oncogénico ${ }^{(1,2)}$. Después de la introducción de la terapia antirretroviral (TARV) en todo el mundo, la tasa de morbilidad y mortalidad debido a las infecciones oportunistas y de algunos cánceres disminuyeron considerablemente ${ }^{(3)}$. Esto produjo que, en los países desarrollados, donde esta terapia está ampliamente disponible, las PVV presenten mayor esperanza de vida y las neoplasias se posicionen como una de las principales causas de muerte ${ }^{(4)}$.

De acuerdo con los criterios de clasificación de los Centros para el Control y la Prevención de Enfermedades (CDC) ${ }^{(5)}$, los cánceres asociados al VIH se clasifican en neoplasias definitorios de sida (NDS) y neoplasias no definitorias de sida (NNDS). Las NDS incluyen el Sarcoma de Kaposi (SK), al linfoma no Hodgkin (LNH) y al cáncer de cérvix invasivo (CCI), todos causados por virus oncogénicos como el virus herpes humano tipo 8 (VHH-8), el virus de Epstein-Barr (VEB) y el virus del papiloma humano (VPH), respectivamente ${ }^{(5)}$. Y las NNDS 
incluyen a todos los tipos de cánceres restantes. Así, pues, desde el amplio uso del TARV, la incidencia de las NDS ha disminuido ampliamente, mientras que el número de NNDS ha aumentado en los últimos años ${ }^{(6,7)}$.

En Perú existe poca evidencia generada en relación con neoplasias en las PVV. De los pocos artículos resaltan los realizados en casos de SK y linfoma de no Hodgkin. Por ejemplo, Mohanna et al. encontraron una incidencia de SK de 20 por 1000 atendidos por VIH entre 1987 y $2003^{(8)}$. Mientras que, en 2018, Cuellar et al. desarrollaron un estudio para determinar los factores pronósticos del linfoma no Hodgkin en pacientes con VIH. Hasta la fecha la información no abarca un estudio que permita describir las características de pacientes VIH que desarrollaron cualquier tipo de cáncer ${ }^{(9)}$. Por lo que, gran parte de la información proviene de estudios realizados en otros países, donde las características epidemiológicas y el contexto de la población son diferentes a la peruana ${ }^{(10-12)}$. Por esa razón, el objetivo de nuestro estudio fue describir y comparar las características demográficas, clínicas, y terapéuticas de las PVV que desarrollaron cáncer en un hospital público de referencia en el Perú.

\section{EL ESTUDIO}

\section{Diseño y población de estudio}

Se realizó un estudio transversal y descriptivo, basado en la información obtenida de los registros de la cohorte de VIH-SIDA (COVIHS), la cual hasta la fecha va enrolando alrededor de 9000 PVV que reciben atención especializada en la Estrategia Sanitaria Nacional de Prevención y Control de ITS, VIH y sida (ESNITSS) en el Hospital Nacional Cayetano Heredia. Se seleccionaron a todas las PVV mayores de 18 años que desarrollaron cualquier tipo de cáncer entre el 1 de enero de 2000 y el 31 de diciembre de 2018, y que estuvieron enroladas en dicha cohorte.

\section{Definición de variables}

Las características demográficas incluyeron la edad al momento del diagnóstico del cáncer, el sexo y el modo de transmisión reportado por el mismo paciente. Las variables relacionadas con el cáncer incluyeron el tipo de cáncer (NDS o NNDS), año calendario de diagnóstico de la neoplasia (período 2000-2004, 2005-2009, 2010-2014 o 2015-2018), y el tiempo desde el diagnóstico de VIH hasta el cáncer. Se tomó en cuenta el diagnóstico de cáncer con un máximo de un año de anterioridad al diagnóstico de VIH, y en cualquier momento después del diagnóstico de VIH ${ }^{(13)}$.

Los valores de laboratorio fueron el nivel de linfocitos CD4 ( $<200$ células/ $\mu \mathrm{L}$ o $\geq 200$ células/ $\mu \mathrm{L})$ y el $\log _{10}$ de la carga viral (suprimido; 2,6-3,9; 4,0-4,9 o $\geq 5,0$ copias $/ \mathrm{mL}$ ), ambos basados en el resultado más reciente registrado entre seis meses antes y un mes después de la fecha de diagnóstico del cáncer ${ }^{(13)}$. El tratamiento para el cáncer comprendió a

\section{MENSAJES CLAVE}

Motivación para realizar el estudio: El riesgo de desarrollar cáncer en las personas que viven con el VIH es mayor que el de la población general. Sin embargo, no existen estudios realizados en Perú.

Principales hallazgos: Las neoplasias no definitorias de sida más frecuentes fueron el sarcoma de Kaposi y el linfoma no Hodgkin; mientras que, entre las neoplasias no definitorias de sida, el linfoma de Hodgkin fue el más común.

Implicancias: Estos hallazgos representan un primer conocimiento sobre cáncer en pacientes con VIH/sida en Perú.

la quimioterapia, radioterapia, cirugía o una combinación entre ellas. No se consideraron las neoplasias intraepiteliales (NIC). Los datos faltantes se mantuvieron y se clasificaron en el grupo desconocido.

\section{Manejo de datos}

La información se obtuvo a través de la extracción autorizada de la base de datos de la COVIHS. Los datos personales como nombres y apellidos estaban asociados a un código numérico. Posteriormente, se creó una nueva base de datos en Excel, respetando los mismos identificadores numéricos con los que trabajó el resto de las variables. Solo los investigadores tuvieron acceso a la base de datos final, y los investigadores principales fueron responsables de su procesamiento y análisis estadístico. Para garantizar la calidad de la información, se verificaron inconsistencias, errores y datos duplicados.

\section{Análisis estadístico}

El análisis descriptivo se resumió utilizando mediana y rango intercuartil (RIC) para variables continuas, y porcentajes para variables categóricas. Para comparar las variables entre los grupos de NDS y NNDS, realizamos la prueba de Chi cuadrado y la prueba de U de Mann-Whitney, según correspondió. Un valor de p menor a 0,05 se consideró estadísticamente significativo. La armonización y el procesamiento de datos se realizó con el paquete estadístico de STATA 14.

\section{Consideraciones éticas}

El protocolo del estudio fue aprobado por el Comité Institucional de Ética de la Universidad Cayetano Heredia. Debido a que la data provino de una base de datos y fue analizada retrospectivamente, se obtuvo exoneración del uso de consentimiento informado. 


\section{HALLAZGOS}

Desde el 2000 al 2018, se identificaron 276 casos de cáncer en 269 PVV, el 80,8\% (223/276) presentaron NDS y el 19,2\% $(53 / 276)$ NNDS. Las NDS más frecuentes fueron el sarcoma de Kaposi con $62,8 \%$ (140/223) y el linfoma no Hodgkin con $30,0 \%$ (67/223); mientras que, entre las NNDS, el linfoma de Hodgkin con 22,6\% (12/53) fue el más común (Tabla 1).

La mediana de edad del total de casos fue de 36,5 años (RIC, 29,7-46,0). El 79,4\% (219/276) fueron hombres. Los hombres que tienen sexo con hombres (HSH) fueron más numerosos en el grupo de las NDS, mientras que los heterosexuales predominaron entre las NNDS. La mediana del tiempo desde el diagnóstico de VIH hasta el cáncer fue de 9,2 meses (RIC, 1,4-52,7); sin embargo, el 77,4\% (41/53) de las NNDS tuvieron un tiempo mayor a 12 meses desde el diagnóstico de VIH hasta el cáncer. La mediana del nivel de linfocitos CD4 fue de 112 células/ $\mu \mathrm{L}$ (RIC, 39,5-253,0), presentando alrededor del 60\% (160/276) niveles debajo de 200 células/ $\mu \mathrm{L}$, y solo el $25,4 \%$ (70/276) en supresión viral al momento del diagnóstico del cáncer (Tabla 2).

A pesar de los datos faltantes en relación con el tratamiento oncológico, el 51,1\% recibió quimioterapia en combinación o como único tratamiento. En el caso de los pacientes con sarcoma de Kaposi no diseminado, el inicio del TARV como tratamiento oncológico fue inmediato al diagnóstico de cáncer. Para el resto de los pacientes con otro tipo de cáncer, la mediana de tiempo desde el diagnóstico de cáncer hasta el tratamiento oncológico sin considerar al TARV fue de 29 días (RIC, 10-72). La Tabla 3 describe los siete pacientes que desarrollaron un segundo cáncer.

Al momento del diagnóstico de cáncer los casos de NNDS presentaron una mediana de edad de 42,2 años y para los casos de NDS una mediana de 35,2 años. La distribución de

Tabla 1. Frecuencias de los tipos de cáncer diagnosticados en el Hospital Cayetano Heredia entre 2000-2018 $(n=276)$

\begin{tabular}{lc}
\hline Tipos de cáncer & N (\%) \\
\hline Neoplasias definitorias de cáncer $(\mathrm{n}=223)$ & $140(62,8)$ \\
Sarcoma de Kaposi & $67(30,0)$ \\
Linfoma no Hodgkin & $16(7,2)$ \\
Cáncer de cérvix invasivo & \\
Neoplasias no definitorias de cáncer $(\mathrm{n}=53)$ & $12(22,6)$ \\
Linfoma Hodgkin & $9(17)$ \\
Cáncer de cérvix in situ & $7(13,2)$ \\
Cáncer de piel & $25(47,2)$ \\
Otras localizaciones & \\
\hline
\end{tabular}

*Incluye estómago, pulmones, ano, recto, ojos, mama, endometrio, ovarios, hígado, próstata, lengua. mujeres a hombres fue de 1:4; sin embargo, entre las NNDS fue de 2:3. La mediana del tiempo desde el diagnóstico de VIH hasta el cáncer entre las NDS fue de 5,8 meses (RIC,1,1-38,1), mientras que entre las NNDS fue de 52,7 meses (RIC,16,8$111,1)$, lo cual demuestra que este último grupo tenía una historia más larga de infección por VIH. Los casos de NDS presentaron niveles de linfocitos CD4 más bajos (mediana de 87,5 células/ $\mu \mathrm{L}$, RIC 37-241) que las NNDS (mediana de 216 células/ $\mu \mathrm{L}$, RIC 129-298), y la carga viral fue indetectable en el 58,5\% (31/53) de las NNDS en comparación con el 17,5\% (39/223) de las NDS. La figura 1 muestra cómo el número de casos de cáncer ha ido aumentado con los años, predominando los casos de NDS sobre las NNDS.

\section{DISCUSIÓN}

Este estudio demuestra que las NDS fueron el grupo de neoplasias más frecuentes entre las PVV, y el SK el tipo de cáncer más común. Las características de las NDS y las NNDS fueron opuestas en casi todas las variables analizadas, por lo que, las NNDS fueron diagnosticadas a una mayor edad, fueron en su mayoría pacientes heterosexuales, tuvieron una historia de VIH más larga, y al momento del diagnóstico de cáncer, niveles de linfocitos CD4 más altos y menores niveles de carga viral.

Estos hallazgos representan un primer conocimiento sobre cáncer en pacientes con VIH-SIDA, ya que hasta la fecha no existen estudios que evalúen conjuntamente a las NDS y las NNDS. A pesar de las limitaciones que implica su naturaleza descriptiva, los resultados no difirieron de otros estudios. Se observaron resultados similares en entornos de ingresos altos y bajos ${ }^{(1,14,15)}$, donde las NDS se encontraron como el grupo predominante; no obstante, algunos últimos reportes de países desarrollados han demostrado que la incidencia de las NNDS ha aumentado ${ }^{(6,7)}$. De acuerdo con informes anteriores ${ }^{(12,14)}$, el SK fue la neoplasia más común entre las NDS, particularmente en lugares donde la coinfección con VHH-8 es alta, como en América Latina y África. Mientras que, entre las NNDS, el LH fue el más frecuente, como se ha visto en otros países ${ }^{(16)}$.

En cuanto a las características demográficas, de acuerdo con otros estudios ${ }^{(17,18)}$, las PVV fueron diagnosticadas de NNDS a una edad más avanzada que las NDS; sin embargo, todavía es una edad muy temprana para desarrollar cáncer en comparación con la población general sin VIH. Similar a un reporto previo ${ }^{(19)}$, los casos en varones fueron más frecuentes respecto a las mujeres; no obstante, algunos estudios realizados en África han demostrado que las frecuencias de cáncer fueron comparables o incluso 
Tabla 2. Características demográficas, clínicas y terapéuticas de los casos diagnosticados con cáncer en el Hospital Cayetano Heredia entre 2000-2018

\begin{tabular}{|c|c|c|c|c|}
\hline \multirow{2}{*}{ Características } & \multirow{2}{*}{$\begin{array}{l}\text { Total } \\
\mathbf{n}(\%) \\
\end{array}$} & \multirow{2}{*}{$\begin{array}{l}\text { NDS } \\
\text { n (\%) } \\
\end{array}$} & \multirow{2}{*}{$\begin{array}{c}\text { NNDS } \\
\mathbf{n}(\%)\end{array}$} & \multirow{2}{*}{ Valor de p } \\
\hline & & & & \\
\hline \multicolumn{5}{|l|}{ Edad } \\
\hline Mediana (RIC) & $36,5(29,7-46,0)$ & $35,2(29,4-43,7)$ & $42,2(32,3-55,6)$ & $0,001^{\mathrm{a}}$ \\
\hline \multicolumn{5}{|l|}{ Sexo } \\
\hline Masculino & $219(79,3)$ & $187(83,9)$ & $32(60,4)$ & $<0,001^{\mathrm{b}}$ \\
\hline Femenino & $57(20,7)$ & $36(16,1)$ & $21(39,6)$ & \\
\hline \multicolumn{5}{|l|}{ Modo de transmisión } \\
\hline Heterosexual & $133(48,2)$ & $95(42,6)$ & $38(71,7)$ & $<0,001^{\mathrm{b}}$ \\
\hline $\mathrm{HSH}$ & $140(50,7)$ & $126(56,5)$ & $14(26,4)$ & \\
\hline Desconocido & $3(1,1)$ & $2(0,9)$ & $1(1,9)$ & \\
\hline \multicolumn{5}{|l|}{ Periodo en años } \\
\hline 2000-2004 & $13(4,7)$ & $11(4,9)$ & $2(3,8)$ & $0,886^{\mathrm{b}}$ \\
\hline 2005-2009 & $53(19,2)$ & $45(20,2)$ & $8(15,1)$ & \\
\hline $2010-2014$ & $96(34,8)$ & $76(34,1)$ & $20(37,7)$ & \\
\hline $2015-2018$ & $114(41,3)$ & $91(40,8)$ & $23(43,4)$ & \\
\hline \multicolumn{5}{|c|}{ Tiempo desde el diagnóstico de VIH hasta el cáncer } \\
\hline Mediana (RIC) & $9,2(1,4-52,7)$ & $5,8(1,1-39,1)$ & $52,7(16,9-99,3)$ & $<0,001^{\mathrm{a}}$ \\
\hline Diagnósticos previos a cáncer & $9(3,3)$ & $6(2,7)$ & $3(5,7)$ & \\
\hline $0-2$ meses & $75(27,2)$ & $71(31,8)$ & $4(7,5)$ & \\
\hline 2-12 meses & $62(22,4)$ & $57(25,6)$ & $5(9,4)$ & \\
\hline$>12$ meses & $130(47,1)$ & $89(39,9)$ & $41(77,4)$ & \\
\hline \multicolumn{5}{|l|}{ Nivel de linfocitos CD4 (células/ $\mu \mathrm{L}$ ) } \\
\hline Mediana (RIC) & $112(39,5-253)$ & $87,5(37-241)$ & $216(129-298)$ & $<0,001^{\mathrm{a}}$ \\
\hline$<200$ & $160(58)$ & $140(62,8)$ & $20(37,7)$ & \\
\hline$\geq 200$ & $84(30,4)$ & $58(26)$ & $26(49,1)$ & \\
\hline Desconocido & $32(11,6)$ & $25(11,2)$ & $7(13,2)$ & \\
\hline \multicolumn{5}{|l|}{ Carga viral (copias/mL) } \\
\hline Mediana (RIC) & $4,7(2,4-5,4)$ & $4,9(3,3-5,5)$ & $1,9(1,6-3,5)$ & $<0,001^{\mathrm{a}}$ \\
\hline Suprimido & $70(25,4)$ & $39(17,5)$ & $31(58,5)$ & \\
\hline $2,6-3,9$ & $29(10,5)$ & $22(9,9)$ & $7(13,2)$ & \\
\hline $4,0-4,9$ & $46(16,7)$ & $41(18,4)$ & $5(9,4)$ & \\
\hline$\geq 5$ & $90(32,6)$ & $87(39)$ & $3(5,7)$ & \\
\hline Desconocido & $41(14,8)$ & $34(15,2)$ & $7(13,2)$ & \\
\hline \multicolumn{5}{|l|}{ Tratamiento oncológico } \\
\hline TARV & $51(18,5)$ & $51(22,9)$ & 0 & $<0,001^{\mathrm{b}}$ \\
\hline Quimioterapia & $124(44,9)$ & $110(49,3)$ & $14(26,4)$ & \\
\hline Radioterapia & $4(1,4)$ & $1(0,4)$ & $3(5,7)$ & \\
\hline Cirugía & $14(5,1)$ & $7(3,2)$ & $7(13,2)$ & \\
\hline $\mathrm{QT}+\mathrm{RT}$ & $14(5,1)$ & $9(4,1)$ & $5(9,4)$ & \\
\hline $\mathrm{QT}+\mathrm{QX}$ & $3(1,1)$ & $1(0,4)$ & $2(3,8)$ & \\
\hline $\mathrm{RT}+\mathrm{QX}$ & $2(0,7)$ & $1(0,4)$ & $1(1,9)$ & \\
\hline Desconocido & $64(23,2)$ & $43(19,3)$ & $21(39,6)$ & \\
\hline
\end{tabular}

NDS: neoplasias definitorias de sida; NNDS: neoplasias no definitorias de sida; RIC: rango intercuartil; HSH: hombres que tiene sexo con hombres; TARV: terapia antirretroviral; QT: quimioterapia; RT: radioterapia; QX: cirugía.

${ }^{\text {a }}$ Prueba U de Mann-Whitney, ${ }^{\text {b }}$ prueba de Chi cuadrado 
Tabla 3. Características de pacientes que desarrollaron un segundo tipo de cáncer diagnosticados en el Hospital Cayetano Heredia entre 2000-2018 $(\mathrm{n}=7)$

\begin{tabular}{lccccc}
\hline Caso & Sexo & $\begin{array}{c}\text { Edad al primer } \\
\text { cáncer }\end{array}$ & Modo de transmisión & Primer cáncer & Segundo cáncer \\
\hline 1 & F & 43 & Heterosexual & CCI & Cáncer de pulmón \\
2 & M & 26 & HSH & SK & LNH \\
3 & M & 38 & Heterosexual & LNH & LNH \\
4 & M & 37 & HSH & LNH & LH \\
5 & M & 30 & HSH & LNH & SK de piel \\
6 & M & 44 & HSH & CCI \\
\hline
\end{tabular}

F: femenino; M: masculino; HSH: hombre que tiene sexo con hombre; CCI: cáncer de cérvix invasivo; SK: sarcoma de Kaposi; LNH: linfoma no Hodgkin; LH: linfoma de Hodgkin

mayores en las mujeres, por la mayor incidencia de VIH entre ellas. Debido a la alta prevalencia de coinfección con VHH-8 entre los HSH, estos presentaron una mayor probabilidad de pertenecer al grupo de las NDS. Por otro lado, aunque en nuestra serie la presencia de segundos cánceres fue baja (siete casos), notamos que la mayoría fueron infecciones relacionadas con VHH-8 y VEB.

Las NNDS también tuvieron una historia más larga de infección por VIH, mayores niveles de linfocitos CD4 y un mayor número de casos en supresión viral que aquellos con NDS. Meijide H. et al. ${ }^{(18)}$, descubrieron que las NNDS tenían un tiempo promedio desde el diagnóstico de VIH hasta el cáncer de 8 años en comparación con las NDS, que tenían un año, así como, alrededor del $70 \%$ de las NNDS presentaron niveles de linfocitos CD4 mayores a 200 células $/ \mu \mathrm{L}$, y casi el $60 \%$ tuvieron una carga viral indetectable. Un segundo estudio realizado en la Ciudad de México por Cornejo-Juárez et al. ${ }^{(20)}$, informaron que casi el $60 \%$ de sus casos presentaron niveles de linfocitos CD4 mayores a 200 células/ $\mu \mathrm{L}$ y una carga viral indetectable. Estos hallazgos

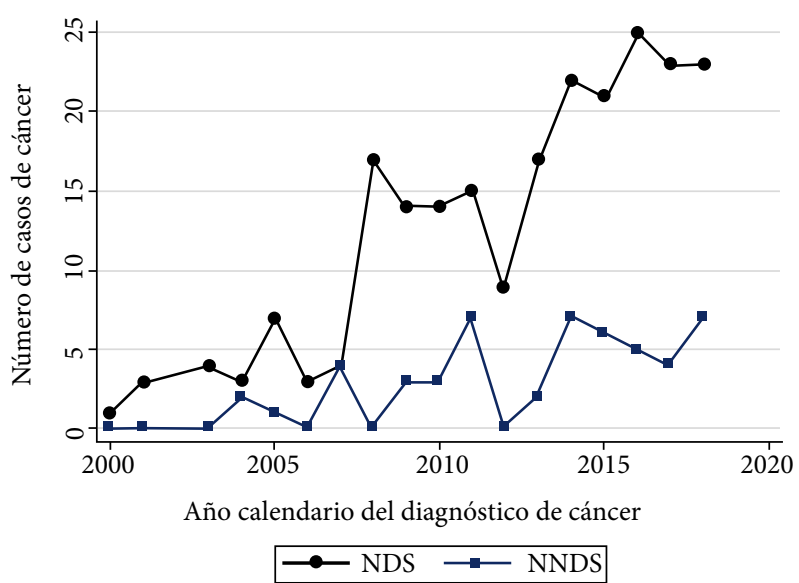

Figura 1. Casos diagnosticados de cáncer según su clasificación en neoplasias definitorias de sida (NDS) y neoplasias no definitorias de sida (NNDS) en el Hospital Cayetano Heredia entre 2000-2018 podrían significar que tanto la inmunosupresión o la supresión viral no tienen un papel importante en el desarrollo de cánceres no relacionados con el sida. Es importante desarrollar estudios de cohorte prospectiva con mayor tamaño muestral y, por ende, mayor potencia; además de incluir variables confusoras para que mediante modelos de regresión podamos establecer si existe asociación entre el diagnóstico de VIH y el desarrollo de NNDS.

La principal limitación se basó en el sesgo de información, ya que se utilizaron los registros de múltiples bases de datos pertenecientes a la cohorte de $\mathrm{VIH}$, los cuales presentan información de diferentes fuentes, como historias clínicas, informes de alta e informes histológicos. De la misma manera, no fue posible recopilar datos sobre algunas variables clínicas que hubieran enriquecido el análisis del estudio, como, por ejemplo, los factores de riesgo tradicionales para cáncer (alcoholismo y tabaquismo), coinfecciones con virus oncogénicos, estadio del cáncer y remisión después del tratamiento. También está incluido el sesgo de selección, ya que se eligieron a todos los pacientes VIH que desarrollaron algún tipo de cáncer y en un solo centro hospitalario.

En conclusión, nuestros hallazgos son similares a otras series de casos en el mundo, donde las NDS fueron el tipo de cáncer más frecuente. Los casos de NNDS presentaron una edad más avanzada, una historia más larga de infección por VIH, niveles de linfocitos CD4 más altos y la mayoría estaban en supresión viral.

Contribuciones de los autores: EGH, FMC Y LMM han participado en la concepción y diseño del artículo. LMM Y JVM realizaron la recolección, análisis e interpretación de datos. LMM y JVM realizaron la redacción del artículo. EGH, FMC y EGL proporcionaron el material de estudio, y participaron en la revisión crítica del contenido del manuscrito. Todos los autores aprobaron la versión final del artículo y asumen la responsabilidad de su contenido.

Financiamiento: Program for Advanced Research Capacities for AIDS in Peru (PARACAS) - 5D43TW009763-05

Conflicto de interés: Los autores declaran no tener conflictos de interés. 


\section{REFERENCIAS BIBLIOGRÁFICAS}

1. Hessol NA, Whittemore H, Vittinghoff E, Hsu LC, Ma D, Scheer S, et al. Incidence of first and second primary cancers diagnosed among people with HIV, 1985-2013: a population-based, registry linkage study. Lancet HIV. 2018;5(11):e647-e655. doi: 10.1016/S2352-3018(18)30179-6

2. Hernández-Ramírez RU, Shiels MS, Dubrow R, Engels EA. Cancer risk in HIV-infected people in the USA from 1996 to 2012: a population-based, registry-linkage study. Lancet HIV. 2017;4(11):e495-e504. doi: 10.1016/ S2352-3018(17)30125-X.

3. Cobucci RN, Lima PH, de Souza PC, Costa VV, Cornetta Mda C, Fernandes JV, et al. Assessing the impact of HAART on the incidence of defining and non-defining AIDS cancers among patients with HIV/ AIDS: a systematic review. J Infect Public Health. 2015;8(1):1-10. doi: 10.1016/j.jiph.2014.08.003.

4. Yarchoan R, Uldrick TS. HIV-Associated Cancers and Related Diseases. N Engl J Med 2018; 378:1029-41. doi: 10.1056/NEJMra1615896

5. Shiels MS, Engels EA. Evolving epidemiology of HIV-associated malignancies. Curr Opin HIV AIDS. 2017;12(1):6-11. doi:10.1097/ COH.0000000000000327

6. Chiu CG, Smith D, Salters KA, Zhang W, Kanters S, Milan D, et al. Overview of cancer incidence and mortality among people living with HIV/AIDS in British Columbia, Canada: Implications for HAART use and NADM development. BMC Cancer. 2017;17(1):270. doi: 10.1186/ s12885-017-3229-1.

7. Brickman C, Palefsky JM. Cancer in the HIV-Infected Host: Epidemiology and Pathogenesis in the Antiretroviral Era. Curr HIV/AIDS Rep. 2015;12(4):388-96. doi: 10.1007/s11904-015-0283-7.

8. Mohanna S, Echaíz J, Ferrufino JC, Bravo F, Gotuzzo E. Perfil clínico y epidemiológico del sarcoma de Kaposi clásico y epidémico: estudio retrospectivo en el Hospital Nacional Cayetano Heredia. Folia dermatol. Peru. 2006; 17 (3): 111-117

9. Cuellar LE, Anampa-Guzmán A, Holguín AM. Velarde J, Portillo-Alvarez D, Zuñiga-Ninquispe MA, et al. Prognostic factors in HIV-positive patients with non-Hodgkin lymphoma: a Peruvian experience. Infect Agents Cancer. 2018;13:27. doi: 10.1186/s13027-018-0200-y

10. Castel AD, Young H, Akiwumi AM, Vargas A, Rogers K, West T, et al. Trends in cancer diagnoses and survival among persons with AIDS in a high HIV prevalence urban area. AIDS Care. 2015;27(7):860-9. doi:10.1 080/09540121.2015.1011074.

11. Hleyhel M, Belot A, Bouvier AM, Tattevin P, Pacanowski J, Genet P, et al. Risk of AIDS-defining cancers among HIV-1-infected patients in France between 1992 and 2009: results from the FHDH-ANRS CO4 cohort. Clin Infect Dis. 2013;57(11):1638-47. doi: 10.1093/cid/cit497

12. Bohlius J, Valeri F, Maskew M, Prozesky H, Garone D, Sengayi M, et al. Kaposis Sarcoma in HIV-infected patients in South Africa: Multicohort study in the antiretroviral therapy era. Int J Cancer. 2014;135(11):26442652. doi:10.1002/ijc.28894

13. Fink VI, Jenkins CA, Castilho JL, Person AK, Shepherd BE, Grinsztejn $\mathrm{B}$, et al. Survival after cancer diagnosis in a cohort of HIV-positive individuals in Latin America. Infect Agent Cancer. 2018;13:16. doi:10.1186/ s13027-018-0188-3

14. Fink VI, Shepherd BE, Cesar C, Krolewiecki A, Wehbe F, Cortés CP, et al. Cancer in HIV-infected persons from the Caribbean, Central and South America. J Acquir Immune Defic Syndr. 2011;56(5):467-473. doi:10.1097/ QAI.0b013e31820bblc3

15. Álvarez-Guevara Deisy, Cuervo-Maldonado Sonia, Sánchez Ricardo, Gómez-Rincón Julio, Ramírez Nancy. Prevalence of defining malignancies in adult patients with HIV/ AIDS in the National Cancer Institute of Colombia. 2007-2014. Rev Fac Med. 2017; 65(3): 397-402. doi: http:// dx.doi.org/10.15446/revfacmed.v65n3.56112

16. Hleyhel M, Bouvier AM, Belot A, Tattevin P, Pacanowski J, Genet P, et al. Risk of non-AIDS-defining cancers among HIV-1-infected individuals in France between 1997 and 2009: results from a French cohort. AIDS. 2014;28(14):2109-18. doi: 10.1097/QAD.0000000000000382.

17. Ramírez-Olivencia G, Valencia-Ortega ME, Martin-Carbonero L, Moreno-Celda V, González-Lahoz J. Malignancies in HIV infected patients: study of 139 cases. Med Clin (Barc). 2009;133(19):729-35. doi: 10.1016/j. medcli.2009.03.043.

18. Meijide Héctor, Mena Alvaro, Pernas Berta, Castro Ángeles, López Soledad, Vázquez Pilar, et al. Neoplasias en pacientes con infección por VIH: Estudio descriptivo de 129 casos en el período 1993-2010. Rev Chil Infectol. 2013; 30(2): 156-161. doi: http://dx.doi.org/10.4067/S071610182013000200006

19. Yang J, Su S, Zhao H, Wang D, Wang J, Zhang F, et al. Prevalence and mortality of cancer among HIV-infected inpatients in Beijing, China. BMC Infect Dis. 2016;16:82. doi:10.1186/s12879-016-1416-3

20. Cornejo-Juárez P, Cavildo-Jerónimo D, Volkow-Fernández P. Non-AIDS defining cancer (NADC) among HIV-infected patients at an oncology tertiary-care center in Mexico. AIDS Res Ther. 2018;15(1):16. doi: 10.1186/ s12981-018-0202-2. 